Research Article

\title{
Reduction of Asymptotic Approximate Expansion of Navier-Stokes Equation and Solution of Inviscid Burgers Equation by Similarity Transformation
}

\author{
Farhad Ali $\left(\mathbb{D},{ }^{1}\right.$ Wali Khan Mashwani $\left(\mathbb{D},{ }^{1}\right.$ Hamayat Ullah, ${ }^{1}$ Ahmed Hussein Msmali $\left(\mathbb{D},{ }^{2}\right.$ \\ Ikramullah Ikramullah $\left(\mathbb{D},{ }^{3}\right.$ and Zabidin Salleh $\mathbb{D}^{4}$ \\ ${ }^{1}$ Institute of Numerical Sciences, Kohat University of Sciences and Technology, Kohat, Pakistan \\ ${ }^{2}$ Department of Mathematics, Collage of Science, Jazan University, Saudi Arabia \\ ${ }^{3}$ Department of Physics, Kohat University of Sciences and Technology, Kohat, Pakistan \\ ${ }^{4}$ Department of Mathematics, Faculty of Ocean Engineering Technology and Informatics, Universiti Malaysia Terengganu, \\ 21030 Kuala Nerus, Terengganu, Malaysia
}

Correspondence should be addressed to Wali Khan Mashwani; mashwanigr8@gmail.com

Received 22 August 2021; Accepted 30 September 2021; Published 16 October 2021

Academic Editor: Punit Gupta

Copyright $\odot 2021$ Farhad Ali et al. This is an open access article distributed under the Creative Commons Attribution License, which permits unrestricted use, distribution, and reproduction in any medium, provided the original work is properly cited.

Symmetry methods for differential equations are a powerful tool for the solutions of differential equations. It linearizes nonlinear differential equations, reduces the order of differential equations, reduces the number of independent variables in partial differential equations, and solves almost all those differential equations for which the other analytic methods fail to solve them. Similarity transformation is a particular case of symmetries, but it is easy and often used to deal with differential equations. The similarity transformation can do all the aforementioned works. In this research, we use the similarity transformation to solve different nonlinear differential equations. Particularly, we will apply this transformation to the nonlinear Navier-Stokes partial differential equations to reduce them to ordinary differential equations. Ordinary differential equations are easy to deal with than partial differential equations. Some nonlinear physical examples of ODEs and PDEs are given to show that the similarity transformation solves those problems where the other analytic methods fail to work.

\section{Introduction}

Differential equations (DEs) are the dynamical equations, which describe the motion of particles. We have a dynamic world, everywhere anytime some dynamic phenomena occur. When mathematicians model a natural phenomenon, they put it in a mathematical system called DEs. DEs are broadly divided into ordinary differential equations (ODEs) and partial differential equations (PDEs). These DEs arise from many purely mathematical considerations, that is, the mathematician defines dependent and independent variables along with parameters corresponding to the given problem and put some constraints accordingly to model and put the given natural phenomenon in a beautiful and elegant short mathematical equation. There is a wide spectrum of different types of DEs, and various techniques and methods have been developed to deal with the quantitative and qualitative behavior of these equations. The field of fluid dynamics, for example, requires different constraints and techniques to model the fluid flow through different media and to make the computation of their solutions possible. The techniques which can handle linear PDEs such as integral transforms and Eigen functions can transform them into ODEs, which is comparably easy to solve. These techniques have some contributions in solving nonlinear PDEs [1]. There are numerous PDE systems that described natural phenomena. Some well-known systems are different types of wave and heat equations, Maxwell equations, Navier-Stokes equations, Schrodinger equation, Dirac equation, etc. 
The majority of the natural phenomena are nonlinear in nature and are therefore modeled into different orders of nonlinear PDEs. Nonlinear PDEs [2, 3] are generally more complex and are therefore difficult to solve using wellestablished analytical and/or numerical techniques. Most of the time, nonlinear PDEs have no exact solutions. In such conditions, in the majority of the cases, we go for their qualitative behavior or at most solve them numerically. It is often very difficult to solve nonlinear PDEs analytically, such as equations of fluid mechanics and equations of plasma dynamics. [4, 5]. However, symmetry methods play an important role to deal with nonlinear DEs (ODEs and PDEs both). The similarity transformation (scaling symmetry) is a way that regroups the existing variables and defines new variables and puts the given harder nonlinear problem in the most simplest and easy form. In most cases, the problem becomes solvable or at least we reduce the order of ODE and the number of independent variables involved in the PDEs. In the case of two variables, PDEs become ODEs. The ODEs are easy to deal with as compared to PDEs.

This method was developed in the early twentieth century by Norwegian mathematician Sophus Lie, who observed that regrouping of variables is applicable in all types of DEs. The solutions obtained through similarity transformation are called similarity solutions, which mostly satisfy simpler equations than the real PDEs. The one-one correspondence between the solution space of the original and new solution space exists, and because of transformation, one can jump back and forth easily. The significance of similarity solutions lies in their simple calculation. We will apply the similarity conversion technique to PDEs to completely solve them or at least simplify them. This transformation decreases the number of autonomous (independent) variables of the system of PDE at least one less than that of the original equation [6]. Hussain et al. [7] recently studied the Hirota-Satsuma coupled system of $\mathrm{KdV}$ equations using OHAM with the addition of Daftardar-Jeffery polynomials. We also employ asymptotic expansion to the reduced Navier-Stokes equation. An asymptotic expansion is the series expansion that has the property of truncation after a finite number of terms and hence gives an approximate solution of the given problem. Power series is the most widely used form of an asymptotic expansion. Integral transforms such as Mellen and Laplace transforms and the Euler-Maclaurin summation formula generate such kinds of expansions. Here in this research, we are going to apply the similarity transformation to solve the nonlinear ODEs and PDEs. Specifically, we are interested in the solution of Burgers and Navier-Stokes equations.

Here in this research, we are going to apply the similarity transformation to solve nonlinear ODEs and PDEs. Specifically, we are interested in the solution of Burgers and Navier-Stokes equations. The organization of the present article goes in the following fashion: basic definitions related to Lie symmetries and similarity transformations are given in Section 2. Subsections 2.1-2.5 show the power of the similarity transformation that how this transformation solves the nonlinear ODEs and PDE. Burgers equations are solved by using appropriate similarity transformations in
Section 3. Section 4 is devoted to the similarity solution of the inviscid Burgers equation. The system of Navier-Stokes equations of fluid dynamics is PDEs. By using the similarity transformations, these equations are converted into a system of ODEs, given in Section 5. The highlights of the investigation are concluded in the final Section 6 .

\section{Basic Definitions}

In this section, we give some basic definitions related to Lie symmetries and solutions of DEs. The similarity symmetry transformation and similarity solution is a special case of the Lie symmetries. Our main concern in this paper is to use the similarity transformation for the solution of DEs. What is more special about the similarity transformation is that one can use it without the knowledge of symmetry methods. That is why we select the similarity transformation among others to handle some nonlinear natural phenomena.

2.1. Lie Symmetries and Their Uses. We have an $n^{\text {th }}$ ordinary differential equation of the form

$$
E\left(x, y, y^{\prime} \ldots y^{n}\right)=0
$$

A transformation,

$$
\begin{aligned}
X & =X(x, y, \varepsilon), \\
X(x, y, 0) & =x, \\
Y & =Y(x, y, \varepsilon), \\
Y(x, y, 0) & =y, \\
x & =x(X, Y, \varepsilon), \\
x(X, Y, 0) & =X, \\
y & =y(X, Y, \varepsilon), \\
y(X, Y, 0) & =Y,
\end{aligned}
$$

is said to be symmetry transformation of equation (1) if it remains invariant under this transformation, that is,

$$
\begin{array}{r}
E\left(x, y, y^{\prime} \ldots y^{n}\right)=E\left(X, Y, Y^{\prime} \ldots Y^{n}\right) \\
=0 .
\end{array}
$$

An $n^{\text {th }}$ order extended Lie symmetry generator is

$$
\begin{aligned}
\mathbf{L}^{[n]}= & \xi(x, y) \frac{\partial}{\partial x}+\eta(x, y) \frac{\partial}{\partial y}+\eta_{x}\left(x, y, y^{\prime}\right) \frac{\partial}{\partial y^{\prime}} \\
& +\cdots+\eta_{\underbrace{x x x \ldots x}_{\text {ntime }}}\left(x, y, y^{\prime} \ldots y^{n}\right) \frac{\partial}{\partial y^{n}},
\end{aligned}
$$

where

$$
\eta_{k}=\frac{\mathrm{d} \eta_{k-1}}{\mathrm{~d} x}-y^{k} \frac{\mathrm{d} \xi}{\mathrm{d} x},
$$

where $k$ is the order of differentiation. If equation (4) is the Lie symmetry generator of the differential equation (1), the Lie symmetry equation takes the form 


$$
\left.\mathbf{L}^{[n]} E\left(x, y, y^{\prime} \ldots y^{n}\right)\right|_{E=0}=0 .
$$

The Lie differential equations corresponding to equation (6) are

$$
\begin{aligned}
\left.\frac{\mathrm{d} X}{\mathrm{~d} \epsilon}\right|_{\epsilon \longrightarrow 0} & =\xi(x, y), \\
X(x, y, 0) & =\left.x \frac{\mathrm{d} Y}{\mathrm{~d} \epsilon}\right|_{\epsilon \longrightarrow 0}=\eta(x, y), \\
Y(x, y, 0) & =y .
\end{aligned}
$$

The integration of Lie's equation gives the corresponding Lie symmetry transformation.

Example 1. Consider the first-order nonlinear ODE

$$
\frac{\mathrm{d} y}{\mathrm{~d} x}=\left(\frac{y^{2}}{x^{3}}\right)+x .
$$

It admits the similarity symmetry generator

$$
\mathbf{L}=x \frac{\partial}{\partial x}+2 y \frac{\partial}{\partial y} .
$$

Using the Lie differential equation, we can find the corresponding Lie symmetry transformation

$$
\begin{aligned}
& X=e^{\epsilon} x, \\
& Y=e^{2 \epsilon} y .
\end{aligned}
$$

The corresponding similarity transformation is

$$
\begin{aligned}
& s=x, \\
& t=\frac{x^{2}}{y} .
\end{aligned}
$$

The differential equation (8) in the similarity variables $s$ and $t$ takes the form

$$
\frac{d s}{d t}=-\frac{1}{(t-1)^{2}},
$$

which is linear, the solution of which is

$$
s=\frac{1}{t-1}+c .
$$

The solution in the original coordinates takes the form

$$
\ln (x)=\frac{y}{\left(x^{2}-y\right)}+c
$$

The ODE given in equation (8) is nonlinear and none of the analytic methods will be applicable here, but we see that the similarity transformation converts it into linear and easily solvable equation. For PDEs, consider a function $v(x, t)$, the dependent and independent variables can be changed by a mapping $(x, t, v) \Longleftrightarrow(X, T, V)$, or more explicitly,

$$
\begin{aligned}
T & =T(t, x, v, \varepsilon), \\
T(t, x, v, 0) & =t \Longleftrightarrow t=t(T, X, V, \varepsilon), \\
t(T, X, V, 0) & =T, \\
X & =X(t, x, v, \varepsilon), \\
X(t, x, v, 0) & =x \Longleftrightarrow x=x(T, X, V, \varepsilon), \\
x(T, X, V, 0) & =X, \\
V & =V(t, x, v, \varepsilon), \\
V(t, x, v, 0) & =v \Longleftrightarrow v=v(T, X, V, \varepsilon), v(T, X, V, 0)=V,
\end{aligned}
$$

where $T, X, V$ are smooth invertible functions of the parameter $\epsilon$ and the given variables $[8,9]$. A PDE is transformed by symmetry transformations given in equation (15), if $V=V(T, X)$ is the solution of given PDE just like the solution $v(t, x)$. In other words, a symmetry transformation maps the solution set of the original PDE to the solution set of the target PDE and there is one correspondence between both the solution sets. Let us explain this by an example.

Example 2. The given PDE

$$
v_{t}(t, x)=v(t, x) v_{x x}(t, x),
$$

which admits the Lie symmetry generator

$$
\mathbf{L}_{p}=t \frac{\partial}{\partial t}+\frac{x}{2} \frac{\partial}{\partial x} \text {. }
$$

The corresponding similarity transformation takes the form

$$
\begin{aligned}
& T=e^{\varepsilon} t, \\
& X=e^{\varepsilon / 2} x, \\
& V=v .
\end{aligned}
$$

Suppose the solution of the given PDE is $v(x, t)$; it is simple to check that $V(T, X)=v\left(e^{\varepsilon} t, e^{\varepsilon / 2} x\right)$ is also the solution simply by putting it into the given PDE, that is,

$$
\begin{aligned}
\partial T V(T, X) & =e^{-\epsilon} \partial_{t} v(T, X) \\
& =V(T, X) \partial_{X X} V(T, X) \\
& =e^{(-\epsilon / 2)-(\epsilon / 2)} v(T, X) \partial_{x x}(v(T, X)) \\
& =e^{-\epsilon} v(T, X) v(T, X) .
\end{aligned}
$$

From the above equation, we cancel the term $e^{-\varepsilon}$ from both sides and get

$$
V(T, X)_{T}=V(T, X) V_{X, X}(T, X) .
$$

Hence, the symmetry transformation given in equation (18) does not change the structure of the given PDE. The PDE given in equation (16) also admit the similarity transformation 


$$
\begin{aligned}
& T=t, \\
& X=\ln (x), \\
& V=\frac{v}{x^{2}} .
\end{aligned}
$$

This transformation reduces the PDE (11) to a first-order ODE

$$
\frac{\mathrm{d} V(T)}{\mathrm{d} T}-2 V^{2}(T)=0,
$$

the solution of which is

$$
V(T)=-\frac{1}{2 T-C}
$$
form

The solution of the PDE given in equation (16) takes the

$$
v(t, x)=-\frac{x^{2}}{2 t-c} .
$$

We see that the PDE (11) is not solvable by another analytic method, but the similarity transformation given in equation (21) easily solved it.

Generally, we can define a similarity transformation for a system of PDEs in $n$ independent variables $\left(x^{1}, x^{2} \ldots x^{n}\right)$ and $m$ dependent variables $u^{j}\left(x^{1}, x^{2} \ldots x^{n}\right), \quad j=$ $1,2,3 \ldots m$, as

$$
\begin{aligned}
& \bar{x}^{i}=e^{\varepsilon a^{i}} x^{i}, \quad i=1,2,3 \ldots n, \\
& \bar{u}^{j}=e^{\varepsilon b^{j}} u^{j}, \quad j=1,2 \ldots m,
\end{aligned}
$$

where $\varepsilon$ is the parameter and $a^{i}, b^{j}$ are constant to be determined correspondingly.

2.2. Similarity Transformation. The transformations which reduce the number of independent variables of a PDE (or a system of PDEs) at least one less than that of the original equation are designated as similarity transformations $[10,11]$.

Example 3. Consider the nonlinear PDE,

$$
u_{t t}(t, x)-u_{x}^{2}(t, x)=0
$$

admits the similarity Lie symmetry (scaling symmetry generator)

$$
\mathbf{L}=t \frac{\partial}{\partial t}-2 u \frac{\partial}{\partial u}
$$

The corresponding similarity transformations is

$$
\begin{aligned}
& T=\ln (t), \\
& X=x, \\
& U=u t^{2} .
\end{aligned}
$$

2.3. Similarity Method. The method which searches for such a similarity transformation is known as similarity method. For example, the transformation given in equation (28) is the similarity transformation corresponding to the PDE given in equation (26).

2.4. Similarity Equations. When we transform a PDE or system of PDEs, $\Omega=0$ (say), in $n$ independent variables to a PDE, $\bar{\Omega}=0$ in $n-1$ independent variables, then the equation is known as similarity equation or similarity representation of the system of $\Omega[12,13]$. Using the similarity transformation given in (19) in the PDE given in equation (26), we have the ordinary differential equation,

$$
\left(U^{\prime}\right)^{2}(X)-6 U(X)=0 .
$$

The similarity transformation given in equation (28) not only reduces the independent variables in the PDE given in (26) but also reduces the order of differentiation. We see that the ODE given in equation (29) is first-order ODE.

2.5. Similarity Solution. The solution which is obtained by employing the similarity transformations is known as similarity solution [14]. The nontrivial similarity solution of the reduced ODE given in equation (29) is

$$
U=\frac{3}{2}\left(X-C_{1}\right)^{2}
$$

When we take the back transformation, the solution in the original coordinates become

$$
u(t, x)=\frac{3}{2}\left(\frac{x-c_{1}}{t}\right)^{2} .
$$

Thus, we solve a nonlinear PDE given in equation (26), with the help of similarity transformation, given in equation (28). The existing analytic methods other than symmetry methods will not be able to solve equation (26).

\section{Solution of Burgers Equation by Similarity Transformation}

Afterwards, we will use the similarity transformation to handle the problems. The detail calculation for finding the similarity transformation corresponding to each PDE is given. There is no need of the symmetry methods' knowledge for finding the similarity transformation. First, we consider the Burgers equation. In the universe, most of the physical phenomena are nonlinear, and the corresponding mathematical models are nonlinear PDEs. Burgers equation is one of the most celebrated PDEs, which models the fluid flow in a dissipative system given by [15-17]

$$
\frac{\partial v(x, t)}{\partial t}+v(x, t) \frac{\partial v(x, t)}{\partial x}=\mu \frac{\partial^{2} v(x, t)}{\partial x^{2}},
$$

where $v(x, t)$ is the velocity field, $x$ is the spatial coordinate, $t$ is the time, and $\mu$ is the viscosity parameter. The term $v_{x x}$ models the diffusion and the $v v_{x}$ models the convective flow, introducing the similarity transformations as 


$$
\begin{aligned}
z_{1} & =\varepsilon^{\alpha} x, \\
s & =\varepsilon^{\beta} t, \\
w & =\varepsilon^{\gamma} v,
\end{aligned}
$$

where $\varepsilon$ is a positive parameter.

As

$$
w=\varepsilon^{\gamma} v,
$$

therefore

$$
\frac{\partial v}{\partial t}=\varepsilon^{\beta-\gamma} \frac{\partial w}{\partial s},
$$

and

$$
\frac{\partial v}{\partial x}=\varepsilon^{\alpha-\gamma} \frac{\partial w}{\partial z_{1}}
$$

Similarly,

$$
\frac{\partial^{2} v}{\partial x^{2}}=\epsilon^{2 \alpha-\gamma} \frac{\partial^{2} w}{\partial z_{1}^{2}}
$$

Putting (35), (36), and (37) in (32), we get

$$
\begin{gathered}
\varepsilon^{\beta-\gamma} \frac{\partial w}{\partial s}+\varepsilon^{-\gamma} w \varepsilon^{\alpha-\gamma} \frac{\partial w}{\partial z_{1}}=\varepsilon^{2 \alpha-\gamma} \frac{\partial^{2} w}{\partial z_{1}^{2}}, \\
\varepsilon^{\beta-\gamma} \frac{\partial w}{\partial s}+w \varepsilon^{\alpha-2 \gamma} \frac{\partial w}{\partial z_{1}}=\varepsilon^{2 \alpha-\gamma} \frac{\partial^{2} w}{\partial z_{1}^{2}} .
\end{gathered}
$$

Equation (38) will be invariant under the similarity transformations, if

$$
\begin{aligned}
\beta-\gamma & =0, \\
\alpha-2 \gamma & =0, \\
2 \alpha-\gamma & =0 .
\end{aligned}
$$

Solving (39), we get

$$
\begin{aligned}
& \frac{\alpha}{\beta}=\frac{1}{2}, \\
& \frac{\gamma}{\beta}=-\frac{1}{2} .
\end{aligned}
$$

So, we take the solution of (47) of the form

$$
\begin{aligned}
v(x, t) & =t^{\gamma / \beta} g(\psi), \\
\psi & =x t^{-(\alpha / \beta)}, \\
v(x, t) & =t^{-(1 / 2)} g(\psi), \\
\psi & =x t^{-(1 / 2) .}
\end{aligned}
$$

Differentiating (41) with respect to $t$, we obtain

$$
v_{t}=-\frac{1}{2} t^{-(3 / 2)} g(\psi)-\frac{1}{2} t^{-(3 / 2)} \psi g^{\prime}(\psi) .
$$

Now, differentiating (41) twice with respect to $x$, we get respectively

$$
\begin{gathered}
v_{x}=t^{-1} g^{\prime}(\psi), \\
v_{x x}=t^{-(3 / 2)} g^{\prime \prime}(\psi) .
\end{gathered}
$$

Putting (41), (42), (43), and(44) in (32), we obtain

$$
\begin{aligned}
& -\frac{1}{2} t^{-(3 / 2)} g(\psi)-\frac{1}{2} t^{-(3 / 2)} \psi g^{\prime}(\psi)+t^{-(1 / 2)} g(\psi) t^{-1} g^{\prime}(\psi) \\
& =t^{-(3 / 2)} g^{\prime \prime}(\psi) .
\end{aligned}
$$

After simplification, we get

$$
g^{\prime \prime}(\psi)+\frac{1}{2} g(\psi)+\frac{1}{2} \psi g^{\prime}(\psi)-g(\psi) g^{\prime}(\psi)=0 .
$$

Equation (46) can be written as

$$
\left(g^{\prime}(\psi)\right)^{\prime}+\left(\frac{\psi}{2} g(\psi)\right)^{\prime}-\frac{1}{2}\left(g^{2}(\psi)\right)^{\prime}=0 .
$$

Integrating (47), we get

$$
g^{\prime}(\psi)+\frac{\psi}{2} g(\psi)-\frac{1}{2} g^{2}(\psi)=m
$$

Equation (48) is the Riccati-type equation, which has standard solution. Its solution is

$$
g(\psi)=\frac{-2 e^{-\psi^{2} / 4}}{C+\sqrt{\pi} e r f(\psi / 2)} .
$$

\section{Solution of Inviscid Burgers Equation by Similarity Transformation}

The inviscid Burgers equation $[18,19]$ for which $\mu=0$ models the nondissipative flow which is given by

$$
\frac{\partial v(x, t)}{\partial t}+v(x, t) \frac{\partial v(x, t)}{\partial x}=0 .
$$

Introducing similarity transformations, we have

$$
\begin{aligned}
z & =\varepsilon^{\alpha} x, \\
s & =\varepsilon^{\beta} t, \\
u & =\varepsilon^{\gamma} v,
\end{aligned}
$$

where $\varepsilon$ is a positive parameter.

As

$$
u=\varepsilon^{\gamma} v
$$

therefore

$$
\frac{\partial v}{\partial t}=\varepsilon^{\beta-\gamma} \frac{\partial u}{\partial s},
$$

and

$$
\frac{\partial v}{\partial x}=\varepsilon^{\alpha-\gamma} \frac{\partial u}{\partial z} .
$$

Putting (53) and (54) in (50), we get 


$$
\varepsilon^{\beta-\gamma} \frac{\partial u}{\partial s}+\varepsilon^{\alpha-2 \gamma} u \frac{\partial u}{\partial z}=0 .
$$

Equation (55) will be invariant under the similarity transformations, if

$$
\gamma=\alpha-\beta
$$

Therefore, we look for the solution of the form

$$
\begin{aligned}
v(x, t) & =t^{\gamma / \beta} g(\phi), \quad \phi=\frac{x}{t^{\alpha / \beta}}, \\
& =t^{(\alpha / \beta)-1} g(\phi) .
\end{aligned}
$$

Let $(\alpha / \beta)-1=m$, then $\alpha / \beta=m+1$; therefore, (25) becomes

$$
v(x, t)=t^{m} g(\phi), \quad \phi=\frac{x}{t^{m+1}} .
$$

Differentiating (58) with respect to $t$, we get

$$
v_{t}=t^{m-1}\left[m g(\phi)-(m+1) \phi g^{\prime}(\phi)\right], \quad \phi=\frac{x}{t^{m+1}} \text {. }
$$

Now, differentiating (58) with respect to $x$, we get

$$
v_{x}(x, t)=t^{m-1} g^{\prime}(\phi) \frac{1}{t^{m}}
$$

Putting (60) and (59) in (54), we obtain

$$
t^{m-1}\left[m g(\phi)-(m+1) \phi g^{\prime}(\phi)\right]+g(\phi) g^{\prime}(\phi)=0 \text {. }
$$

For $m=0$, (61) becomes

$$
t^{-1}\left[-\phi g^{\prime}(\phi)+g(\phi) g^{\prime}(\phi)\right]=0
$$

since $t \neq 0$, therefore

$$
-\phi g^{\prime}(\phi)+g(\phi) g^{\prime}(\phi)=0
$$

This gives

$$
g(\phi)=\phi
$$

Therefore,

$$
g(\phi)=\frac{x}{t^{\alpha / \beta}}, \quad \phi=\frac{x}{t^{\alpha / \beta}}
$$

As $(\alpha / \beta)-1=m$, but $m=0$, therefore $\alpha / \beta=1$. Now, equation (64) becomes

$$
g(\phi)=\frac{x}{t}
$$

so

$$
v(x, t)=t^{m} g(\phi) .
$$

Putting $m=0$ and $g(\phi)=x / t$, we have

$$
v(x, t)=\frac{x}{t}
$$

which is a solution of the inviscid Burgers equation.

\section{Reduction of Navier-Stokes Equations by Similarity Transformations}

Fluid dynamics is a hot area of research and almost all dynamical equations that describe the motion of fluids are nonlinear. The basic ingredients of the fluid dynamics are the Navier-Stokes equations of fluid motion. The Navier-Stokes equations are nonlinear in nature and thus it is very difficult to find their exact/analytical solutions. Therefore, different constraint/assumptions are needed to approach the possible solutions of these equations. The analytic solution of the Navier-Stokes equations is possible only if we ignore the complexities and nonlinearities in the equations, or we proceed toward the possible solutions with numerical computation. But, these types of solutions not provide the actual picture of problem. The general compact form of the Navier-Stokes equations is

$$
\begin{aligned}
\frac{\mathrm{d} \rho}{\mathrm{d} t}+\rho(\nabla \cdot \mathbf{V}) & =0, \\
\rho \frac{\mathrm{d} \mathbf{V}}{\mathrm{d} t} & =\rho \mathbf{g}-\nabla P+\mu \nabla^{2} \mathbf{V} .
\end{aligned}
$$

where $\rho(t)$ is the fluid density, $\mathbf{V}=(u, v, w)$ is the fluid velocity, $\mathbf{g}=\left(g_{x}, g_{y}, g_{z}\right)$ is the gravitational acceleration, $P$ is the pressure of fluid, $\mu$ is the a constant, and

$$
\begin{aligned}
& \nabla=\frac{\partial}{\partial x} \mathbf{i}+\frac{\partial}{\partial y} \mathbf{j}+\frac{\partial}{\partial z} \mathbf{k}, \\
& \frac{\mathrm{d}}{\mathrm{d} t}=\frac{\partial}{\partial t}+\dot{x}^{i} \frac{\partial}{\partial x^{i}}, \quad i=1,2,3 .
\end{aligned}
$$

In more explicit form, the Navier-Stokes equation given (69) can be written as 


$$
\begin{aligned}
\frac{d \rho}{d t}+\rho\left(\frac{\partial u}{\partial x}+\frac{\partial v}{\partial y}+\frac{\partial w}{\partial z}\right) & =0 \\
\mathbf{i}: \rho\left(\frac{\partial u}{\partial t}+u \frac{\partial u}{\partial x}+v \frac{\partial u}{\partial y}+w \frac{\partial u}{\partial z}\right) & =\rho g_{x}-\frac{\partial P}{\partial x}+\mu\left(\frac{\partial^{2} u}{\partial x^{2}}+\frac{\partial^{2} u}{\partial y^{2}}+\frac{\partial^{2} u}{\partial z^{2}}\right) \\
\mathbf{j}: \rho\left(\frac{\partial v}{\partial t}+u \frac{\partial v}{\partial x}+v \frac{\partial v}{\partial y}+w \frac{\partial v}{\partial z}\right) & =\rho g_{y}-\frac{\partial P}{\partial y}+\mu\left(\frac{\partial^{2} v}{\partial x^{2}}+\frac{\partial^{2} v}{\partial y^{2}}+\frac{\partial^{2} v}{\partial z^{2}}\right) \\
\mathbf{k}: \rho\left(\frac{\partial w}{\partial t}+u \frac{\partial w}{\partial x}+v \frac{\partial w}{\partial y}+w \frac{\partial w}{\partial z}\right) & =\rho g_{z}-\frac{\partial P}{\partial z}+\mu\left(\frac{\partial^{2} w}{\partial x^{2}}+\frac{\partial^{2} w}{\partial y^{2}}+\frac{\partial^{2} w}{\partial z^{2}}\right)
\end{aligned}
$$

For the steady flow of the fluid, the flow becomes time independent, in that case with $\mathrm{d} / \mathrm{d} t$ and $\partial / \partial t$ becoming zero, that is, the system (73) takes the form

$$
\begin{aligned}
\frac{\partial u}{\partial x}+\frac{\partial v}{\partial y}+\frac{\partial w}{\partial z} & =0 \\
\mathbf{i}: \rho\left(u \frac{\partial u}{\partial x}+v \frac{\partial u}{\partial y}+w \frac{\partial u}{\partial z}\right) & =\rho g_{x}-\frac{\partial P}{\partial x}+\mu\left(\frac{\partial^{2} u}{\partial x^{2}}+\frac{\partial^{2} u}{\partial y^{2}}+\frac{\partial^{2} u}{\partial z^{2}}\right) \\
\mathbf{j}: \rho\left(u \frac{\partial v}{\partial x}+v \frac{\partial v}{\partial y}+w \frac{\partial v}{\partial z}\right) & =\rho g_{y}-\frac{\partial P}{\partial y}+\mu\left(\frac{\partial^{2} v}{\partial x^{2}}+\frac{\partial^{2} v}{\partial y^{2}}+\frac{\partial^{2} v}{\partial z^{2}}\right) \\
\mathbf{k}: \rho\left(u \frac{\partial w}{\partial x}+v \frac{\partial w}{\partial y}+w \frac{\partial w}{\partial z}\right) & =\rho g_{z}-\frac{\partial P}{\partial z}+\mu\left(\frac{\partial^{2} w}{\partial x^{2}}+\frac{\partial^{2} w}{\partial y^{2}}+\frac{\partial^{2} w}{\partial z^{2}}\right)
\end{aligned}
$$

For incompressible fluid, the density of the fluid becomes independent of time, therefore we have $\mathrm{d} \rho / \mathrm{d} t=0$. In that case, we have

$$
\begin{gathered}
\frac{\partial u}{\partial x}+\frac{\partial v}{\partial y}+\frac{\partial w}{\partial z}=0 \\
\mathbf{i}: \rho\left(\frac{\partial u}{\partial t}+u \frac{\partial u}{\partial x}+v \frac{\partial u}{\partial y}+w \frac{\partial u}{\partial z}\right)=\rho g_{x}-\frac{\partial P}{\partial x}+\mu\left(\frac{\partial^{2} u}{\partial x^{2}}+\frac{\partial^{2} u}{\partial y^{2}}+\frac{\partial^{2} u}{\partial z^{2}}\right) \\
\mathbf{j}: \rho\left(\frac{\partial v}{\partial t}+u \frac{\partial v}{\partial x}+v \frac{\partial v}{\partial y}+w \frac{\partial v}{\partial z}\right)=\rho g_{y}-\frac{\partial P}{\partial y}+\mu\left(\frac{\partial^{2} v}{\partial x^{2}}+\frac{\partial^{2} v}{\partial y^{2}}+\frac{\partial^{2} v}{\partial z^{2}}\right) \\
\mathbf{k}: \rho\left(\frac{\partial w}{\partial t}+u \frac{\partial w}{\partial x}+v \frac{\partial w}{\partial y}+w \frac{\partial w}{\partial z}\right)
\end{gathered}
$$

We consider the incompressible fluid in the $x y$ plane, which flows steadily in the $x$ direction [20-22]. For the asymptotic behavior of the fluid flow, an asymptotic term $U(x)(\mathrm{d} U / \mathrm{d} x)=$ $\rho g_{x}-\partial P / \partial x$ is included in the dynamics which satisfy the given conditions. Our first aim is to find and use the similarity transformation and reduce the given nonlinear Navier-Stokes PDEs equations in ODEs equations. A step-by-step procedure for this conversion is given in the remainder of this paper. 


$$
\begin{aligned}
\frac{\partial u}{\partial x}+\frac{\partial v}{\partial y} & =0, \\
u \frac{\partial u}{\partial x}+v \frac{\partial u}{\partial y} & =\frac{\partial^{2} u}{\partial y^{2}}+U \frac{\mathrm{d} U}{\mathrm{~d} x}, \\
u(x, 0) & =0, \\
v(x, 0) & =0, \\
u(x, \infty) & =U(x) .
\end{aligned}
$$

Introducing the following similarity transformation,

$$
\begin{aligned}
x^{\prime} & =e^{\varepsilon a_{1}} x, \\
y^{\prime} & =e^{\varepsilon b_{1}} y, \\
u^{\prime} & =e^{\varepsilon c_{1}} u, \\
v^{\prime} & =e^{\varepsilon p_{1}} v, \\
U^{\prime} & =e^{\varepsilon q_{1}} U,
\end{aligned}
$$

from (75), we have

$$
\begin{aligned}
& \frac{\partial u}{\partial x}=e^{\varepsilon\left(a_{1}-c_{1}\right)} \frac{\partial u^{\prime}}{\partial x^{\prime}} . \\
& \frac{\partial u}{\partial y}=e^{\varepsilon\left(b_{1}-c_{1}\right)} \frac{\partial u^{\prime}}{\partial y^{\prime}} \\
& \frac{\partial v}{\partial y}=e^{\epsilon\left(b_{1}-p_{1}\right)} \frac{\partial v^{\prime}}{\partial y^{\prime \prime}} \\
& \frac{\partial^{2} u}{\partial y^{2}}=e^{\epsilon\left(2 b_{1}-c_{1}\right)} \frac{\partial u^{\prime}}{\partial y^{\prime}}, \\
& \frac{\mathrm{d} U}{\mathrm{~d} x}=e^{\epsilon\left(a_{1}-q_{1}\right)} \frac{\mathrm{d} U^{\prime}}{\mathrm{d} x^{\prime}} .
\end{aligned}
$$
get

Putting the above equations from (76) to (80) in (74), we

$$
\begin{aligned}
e^{\varepsilon\left(a_{1}-c_{1}\right)} \frac{\partial u^{\prime}}{\partial x^{\prime}}+e^{\varepsilon\left(b_{1}-p_{1}\right)} \frac{\partial v^{\prime}}{\partial y^{\prime}} & =0, \\
e^{\varepsilon\left(a_{1}-2 c_{1}\right)} u^{\prime} \frac{\partial u^{\prime}}{\partial x^{\prime}}+e^{\varepsilon\left(b_{1}-c_{1}-p_{1}\right)} v^{\prime} \frac{\partial u^{\prime}}{\partial y^{\prime}}= & e^{\varepsilon\left(2 b_{1}-c_{1}\right)} \frac{\partial^{2} u^{\prime}}{\partial y^{\prime 2}} \\
& +e^{\varepsilon\left(a_{1}-2 q_{1}\right)} \frac{\mathrm{d} U^{\prime}}{\mathrm{d} x^{\prime}}, \\
u^{\prime}\left(x^{\prime}, 0\right)= & 0, \\
v^{\prime}\left(x^{\prime}, 0\right) & =0, \\
u^{\prime}\left(x^{\prime}, \infty\right) & =U(x) .
\end{aligned}
$$

In (81), dividing by the coefficients of the leading terms in each equation, we get the transformed equations as

$$
\begin{aligned}
\frac{\partial u^{\prime}}{\partial x^{\prime}}+e^{\epsilon\left(b_{1}+c_{1}-a_{1}-p_{1}\right)} \frac{\partial v^{\prime}}{\partial y^{\prime}} & =0, \\
u^{\prime} \frac{\partial u^{\prime}}{\partial x^{\prime}}+e^{\epsilon\left(b_{1}+c_{1}-a_{1}-p_{1}\right)} v^{\prime} \frac{\partial u^{\prime}}{\partial y^{\prime}} & =e^{\epsilon\left(2 b_{1}+c_{1}-a_{1}\right)} \frac{\partial^{2} u^{\prime}}{\partial y^{\prime 2}} \\
& +e^{2 \epsilon\left(c_{1}-q_{1}\right)} U^{\prime} \frac{\mathrm{d} U^{\prime}}{\mathrm{d} x^{\prime}}, \\
u^{\prime}\left(x^{\prime}, 0\right) & =0, \\
v^{\prime}\left(x^{\prime}, 0\right) & =0, \\
u^{\prime}\left(x^{\prime}, \infty\right) & =e^{\epsilon\left(c_{1}-q_{1}\right)} U(x) . \\
b_{1}+c_{1}-a_{1}-p_{1} & =0, \\
2 b_{1}+c_{1}-a_{1} & =0, \\
c_{1}-q_{1} & =0 .
\end{aligned}
$$

Solving (83), we get

$$
\begin{aligned}
& p_{1}=\frac{\left(c_{1}-a_{1}\right)}{2}, \\
& b_{1}=\frac{\left(a_{1}-c_{1}\right)}{2}, \\
& c_{1}=q_{1} .
\end{aligned}
$$

Now, we have two arbitrary parameters $a_{1}$ and $c_{1}$; this gives flexibility in assigning specific values to these parameters. We assume that $c_{1}=r a_{1}$, where $r$ is another parameter. Using this new parameter, (84) becomes

$$
\begin{aligned}
& p_{1}=\frac{\left(r a_{1}-a_{1}\right)}{2}, \\
& b_{1}=\frac{\left(a_{1}-r a_{1}\right)}{2}, \\
& q_{1}=r a_{1} .
\end{aligned}
$$

Expanding the exponentials in (75) by Taylor series and keeping the terms up to first order in $\epsilon$ and denoting the difference in transformed and original variables as differential, we have for the first term,

$$
\begin{aligned}
x^{\prime} & =e^{\varepsilon a_{1}} x, \\
x^{\prime} & =\left(1+\varepsilon a_{1}\right) x, \\
x^{\prime} & =x+a_{1} \varepsilon x, \\
x^{\prime}-x & =a_{1} \varepsilon x, \\
\mathrm{~d} x & =a_{1} \varepsilon x, \\
\frac{\mathrm{d} x}{a_{1} x} & =\varepsilon .
\end{aligned}
$$


Similarly, the expansion of the other terms gives

$$
\begin{aligned}
\frac{\mathrm{d} y}{b_{1} y} & =\varepsilon . \\
\frac{\mathrm{d} u}{c_{1} u} & =\varepsilon . \\
\frac{\mathrm{d} v}{p_{1} v} & =\varepsilon . \\
\frac{\mathrm{d} U}{q_{1} U} & =\varepsilon .
\end{aligned}
$$

Comparing (86), (87), (88), (89), and (90), we have

$$
\begin{aligned}
\frac{\mathrm{d} x}{a_{1} x} & =\frac{\mathrm{d} y}{b_{1} y} \\
& =\frac{\mathrm{d} u}{c_{1} u} \\
& =\frac{\mathrm{d} v}{p_{1} v} \\
& =\frac{\mathrm{d} U}{q_{1} U} .
\end{aligned}
$$
obtain

Using the values of $b_{1}, c_{1}, p_{1}$, and $q$ from (85) in (91), we

$$
\begin{aligned}
\frac{\mathrm{d} x}{x} & =\frac{\mathrm{d} y}{((1-r) / 2) y} \\
& =\frac{\mathrm{d} u}{r u} \\
& =\frac{\mathrm{d} v}{((r-1) / 2) v} \\
& =\frac{\mathrm{d} U}{r U} .
\end{aligned}
$$

Using the method of characteristic to solve (92), the solution of

$$
\frac{\mathrm{d} x}{x}=\frac{\mathrm{d} y}{((1-r) / 2) y},
$$

is

$$
\phi=y x^{(r-1) / 2} .
$$

Similarly, the solutions of the other equations in (92) are respectively given by

$$
\begin{aligned}
& u=x^{r} f(\phi), \\
& v=x^{(r-1) / 2} g(\phi), \\
& U=k x^{r} .
\end{aligned}
$$

Thus, we obtained the following similarity variable and functions:

$$
\begin{aligned}
\phi & =y x^{(r-1) / 2}, \\
u & =x^{r} f(\phi), \\
v & =x^{(r-1) / 2} g(\phi), \\
U & =k x^{r},
\end{aligned}
$$

where $k$ is constant, using (97) to obtain the first- and second-order derivatives as in the following,

$$
\begin{aligned}
\frac{\partial u}{\partial x} & =r x^{r-1} f(\phi)+\frac{r-1}{2} x^{r-1} \phi f^{\prime}(\phi), \\
\frac{\partial u}{\partial y} & =x^{r} \cdot x^{r-1 / 2} f^{\prime}(\phi), \\
\frac{\partial^{2} u}{\partial y^{2}} & =x^{r} \cdot x^{r-1} f^{\prime \prime}(\phi), \\
\frac{\partial v}{\partial y} & =x^{(r-1)} g^{\prime}(\phi) .
\end{aligned}
$$

Using these derivatives in (74), we get

$$
\begin{gathered}
r f(\phi)+\frac{r-1}{2} \phi f^{\prime}(\phi)+g^{\prime}(\phi)=0, \\
\left(\frac{r-1}{2}\right) \phi f(\phi) f^{\prime}(\phi)+r f^{2}(\phi)+g(\phi) f^{\prime}(\phi)=f^{\prime \prime}(\phi)+r k .
\end{gathered}
$$

We have reduced the Navier-Stokes equation,

$$
\begin{aligned}
\frac{\partial u}{\partial x}+\frac{\partial v}{\partial y} & =0, \\
u \frac{\partial u}{\partial x}+v \frac{\partial u}{\partial y} & =\frac{\partial^{2} u}{\partial y^{2}}+U \frac{\mathrm{d} U}{\mathrm{~d} x}, \\
u(x, 0) & =0, \\
v(x, 0) & =0, \\
u(x, \infty) & =U(x),
\end{aligned}
$$

to ODEs using the proposed similarity transformations

$$
r f(\phi)+\frac{r-1}{2} \phi f^{\prime}(\phi)+g^{\prime}(\phi)=0,
$$

$$
\begin{aligned}
\left(\frac{r-1}{2}\right) \phi f(\phi) f^{\prime}(\phi)+r f^{2}(\phi)+g(\phi) f^{\prime}(\phi) & =f^{\prime \prime}(\phi)+r k, \\
f(0) & =0, \\
g(0) & =0, \\
f(M) & =k, \\
M & \longrightarrow \infty .
\end{aligned}
$$




\section{Conclusion}

In this research, we used the similarity transformation to solve completely or at least reduce the nonlinear PDEs to nonlinear ODEs. As the similarity transformations are the subclass of Lie symmetries, therefore some basic definitions related to Lie symmetry along with examples are given in Section 2. In the same section, we see the power of similarity transformation in solving the nonlinear ODEs and PDEs. Similarity transformation is a technique for solving complex and nonlinear DEs without the knowledge of Lie symmetry methods, that is, the main reason for using the similarity transformation in this research work. In this method, one takes a general similarity transformation, inserts it into the given equation (or system of equations), and solves for a particular similarity transformation. This procedure is shown in detail in Sections 3 and 4.

Generally, modeling fluid flow is a complex phenomenon due to its nonlinear nature. Burgers equation models the diffusion of the viscous fluid, whereas the inviscid Burgers equation is its special case by ignoring the viscous nature of the fluid. The mathematical model which described Burgers equation is nonlinear and cannot be solved analytically. We solve Burgers equation by using the similarity transformations. This technique reduced the number of independent variables in the equation and reduced the nonlinear PDEs to nonlinear ODEs. The similarity transformation transforms Burgers equation into Riccati-type equation, which has a standard solution. The detailed and step-by-step calculation of this work is given in Section 3.

The Navier-Stokes equations are second-order nonlinear PDEs that had been developed to model the compressible viscous fluid flow by adding the viscous term to the original Euler equations, which models the compressible friction-less flow. The general Navier-Stokes equations deal with compressible, viscous, nonsteady, and steady flow fluids. One can simplify these equations for his/her problem nature. For example, most of the fluids are incompressible, which implies that the density of the fluids does not depend on time, and equations become independent of the density derivative term. Similarly, for a steady flow, the velocity of the fluids becomes independent of time, which not only simplifies the equations but also helps in solving these nonlinear PDEs. The similarity transformation corresponding to the Navier-Stokes equations is given in Section 4. We reduced the nonlinear system of PDEs of the Navier-Stokes equations to nonlinear ODEs by using the similarity transformation. The quantitative and qualitative analyses of the transformed ODE system are the future works in which we will give the complete analysis of the fluid dynamics of the Navier-stokes equations.

\section{Data Availability}

No specific data were used to support this study.

\section{Conflicts of Interest}

The authors declare that they have no conflicts of interest to report regarding the present study.

\section{References}

[1] S. Dubljevic and P. D. Christofides, "Predictive output feedback control of parabolic partial differential equations (PDEs)," Industrial \& Engineering Chemistry Research, vol. 45, no. 25, pp. 8421-8429, 2006.

[2] E.-G. Fan, "'Traveling wave solutions for nonlinear equations using symbolic computation," Computers \& Mathematics with Applications, vol. 43, no. 6-7, pp. 671-680, 2002.

[3] G. Adomian, "A new approach to nonlinear partial differential equations," Journal of Mathematical Analysis and Applications, vol. 102, no. 2, pp. 420-434, 1984.

[4] D. A. Tarzia, "'A bibliography on moving-free boundary problems for the heat-diffusion equation," The Stefan and related problems, MAT-Serie A, vol. 2, 2000.

[5] J. Hristov, "An approximate analytical (integral-balance) solution to a nonlinear heat diffusion equation," Thermal Science, vol. 19, no. 2, pp. 723-733, 2015.

[6] M. Ali Aroon and M. A. Khansary, "Generalized similarity transformation method applied to partial differential equations (PDEs) in falling film mass transfer," Computers \& Chemical Engineering, vol. 101, pp. 73-80, 2017.

[7] K. Zawar Hussain, S. Khan, A. Ullah et al., "Extension of optimal homotopy asymptotic method with use of Daftardar-Jeffery polynomials to Hirota-Satsuma coupled system of Korteweg-de Vries equations," Open Physics, vol. 18, no. 6, pp. 916-924, 2020.

[8] B. Fornberg and C. Piret, "A stable Algorithm for flat radial basis functions on a sphere," SIAM Journal on Scientific Computing, vol. 30, no. 1, pp. 60-80, 2008.

[9] C. Sophocleous, "Transformation properties of a variablecoefficient Burgers equation," Chaos, Solitons \& Fractals, vol. 20, no. 5, pp. 1047-1057, 2004.

[10] Y. Cho and A. Aessopos, "Similarity transformation methods in the analysis of the two dimensional steady compressible laminar boundary layer," Term paper, vol. 2, 2004.

[11] J. V. Lambers, "Derivation of high-order spectral methods for time-dependent PDE using modified moments," Electronic Transactions on Numerical Analysis, vol. 28, pp. 114-135, 2008.

[12] S.-y. Lou, D.-f. Hang-yu-Ruan, D.-f. Chen, and W.-z. Chen, "Similarity reductions of the KP equation by a direct method," Journal of Physics A: Mathematical and General, vol. 24, no. 7, pp. 1455-1467, 1991.

[13] S.-J. Liao, "A general approach to get series solution of nonsimilarity boundary-layer flows," Communications in Nonlinear Science and Numerical Simulation, vol. 14, no. 5, pp. 2144-2159, 2009.

[14] E. C. Dauenhauer and J. Majdalani, "Exact self-similarity solution of the Navier-Stokes equations for a porous channel with orthogonally moving walls," Physics of Fluids, vol. 15, no. 6, pp. 1485-1495, 2003.

[15] J. Doyle and M. J. Englefield, "Similarity solutions of a generalized burgers equation," IMA Journal of Applied Mathematics, vol. 44, no. 2, pp. 145-153, 1990.

[16] E. Y. Rodin, "A Riccati solution for Burgers' equation," Quarterly of Applied Mathematics, vol. 27, no. 4, pp. 541-545, 1970.

[17] M. Nadjafikhah, "Classification of similarity solutions for inviscid burgers' equation," Advances in Applied Clifford Algebras, vol. 20, no. 1, pp. 71-77, 2010.

[18] E. R. Benton and G. W. Platzman, "A table of solutions of the one-dimensional Burgers equation," Quarterly of Applied Mathematics, vol. 30, no. 2, pp. 195-212, 1972. 
[19] M. Nadjafikhah, "Lie symmetries of inviscid burgers' equation," Advances in Applied Clifford Algebras, vol. 19, no. 1, pp. 101-112, 2009.

[20] H. Xu, Z.-L. Lin, S.-J. Liao, J.-Z. Wu, and J. Majdalani, "Homotopy based solutions of the Navier-Stokes equations for a porous channel with orthogonally moving walls," Physics of Fluids, vol. 22, no. 5, Article ID 053601, 2010.

[21] P. Lagerstrom and J. Cole, "Examples illustrating expansion procedures for the Navier-Stokes equations," Indiana University Mathematics Journal, vol. 4, no. 6, pp. 817-882, 1955.

[22] K. O. Friedrichs, "Asymptotic phenomena in mathematical physics," Bulletin of the American Mathematical Society, vol. 61, no. 6, pp. 485-505, 1955. 